\title{
Gene Therapy with Cytosine Deaminase and Endostatin Fusion Gene Mediated by Endothelial Progenitor Cells in Hepatomas
}

This article was published in the following Dove Press journal: Cancer Management and Research

Yue-Lin Zhang (iD $^{1,2, *}$ Tan-Yang Zhou ${ }^{1,2, *}$ Jing $\mathrm{Ai}^{3, *}$ Sheng-Qun Chen ${ }^{1,2, *}$ Feng Chen (ID) Chun-Hui $\mathrm{Nie}^{1,2}$ Xin-Hua Chen (D) ${ }^{1,2}$ Guan-Hui Zhou (iD) ${ }^{1,2}$ Hong-Liang Wang ${ }^{1,2}$ TongYin Zhu ${ }^{1,2}$ Bao-Quan Wang ${ }^{1,2} \mathrm{Zi}-\mathrm{Niu}$ $\mathrm{Yu}^{1,2}$ Li Jing ${ }^{1,2}$ Li-Ming Wu ${ }^{1,2}$ Shu-Sen Zheng ${ }^{1,2}$ Jun-Hui Sun ${ }^{1,2}$

'Hepatobiliary and Pancreatic Interventional Treatment Center, Division of Hepatobiliary and Pancreatic Surgery, The First Affiliated Hospital, Zhejiang University School of Medicine, Hangzhou 310003, Zhejiang Province, People's Republic of China; ${ }^{2} \mathrm{Key}$ Laboratory of Combined Multi-Organ Transplantation, Ministry of Public Health, Hangzhou 310003, Zhejiang Province, People's Republic of China; ${ }^{3}$ Eye Centre, The Second Affiliated Hospital, Zhejiang University School of Medicine, Hangzhou 310009, Zhejiang Province, People's Republic of China; ${ }^{4}$ Department of Radiology, The First Affiliated Hospital, Zhejiang University School of Medicine, Hangzhou 310003, Zhejiang Province, People's Republic of China

*These authors contributed equally to this work

Correspondence: Jun-Hui Sun Hepatobiliary and Pancreatic Interventional Treatment Center, Division of Hepatobiliary and Pancreatic Surgery, The First Affiliated Hospital, Zhejiang University School of Medicine; Zhejiang Clinical Research Center of Hepatobiliary and Pancreatic Diseases, 79 Qingchun Road, Hangzhou 310003, Zhejiang

Province, People's Republic of China

Tel +8657187236815

Email 1307005@zju.edu.cn

Feng Chen

Department of Radiology, The First Affiliated Hospital, Zhejiang University School of Medicine, 79 Qingchun Road, Hangzhou 310003, Zhejiang Province,

People's Republic of China

Tel +8657187236815

Email chenfenghz@zju.edu.cn
Purpose: Gene-targeting therapy provides a novel therapeutic approach for tumor treatment using genetically modified endothelial progenitor cells (EPCs) as cellular carriers. This study applied EPCs armed with cytosine deaminase (CD) and endostatin (ES) fusion gene in liver cancer to explore its therapeutic effect.

Materials and Methods: EPCs from heart blood of male BALB/c nude mice were cultured and transfected with CD and ES fusion gene. Subsequently, these genetically modified cells were injected into mice bearing hepatoma through their tail veins. The tumor volumes and cell apoptosis were followed up.

Results: Tumor volume in the group injected CD/ES-EPCs greatly decreased. The positive rate of VEGF and CD31 in the tumor tissue was lowest in the CD/ES-EPC group. Furthermore, the number of apoptotic cells was highest in the CD/ES-EPC group.

Conclusion: The EPCs transfected with $\mathrm{CD} / \mathrm{ES}$ inhibited tumor growth and preferentially induced tumor cell apoptosis, providing a novel methodology for cancer-targeting therapy.

Keywords: gene-targeting therapy, endothelial progenitor cell, endostatin, cytosine deaminase, hepatoma, transfection

\section{Introduction}

Hepatoma is the second leading cause of cancer-related deaths and one of the most common malignant tumors worldwide. ${ }^{1,2}$ Although many surgical and nonsurgical treatments are available, eg liver transplantation, resection, transarterial chemoembolization, radio frequency ablation, and percutaneous ethanol injections, ${ }^{3}$ the longterm prognosis remains poor. ${ }^{4-6}$ Therefore, researchers are working on novel therapeutic approaches to improve the treatment outcomes of hepatoma patients. ${ }^{7}$ Currently, gene-targeting therapy is potential candidate that can selectively attack the cancer by genetically-modified EPCs as cellular vectors. ${ }^{8}$

Endothelial progenitor cells (EPCs) play a decisive role in tumor vasculogenesis. Accumulating evidence indicates that EPCs migrate from the bone marrow into tumor tissues in vivo and differentiate into a new endothelium in the tumor bed, inducing the formation of new blood vessels. ${ }^{9-11}$ EPCs can be used as cellular vehicles for cancer therapy. ${ }^{8}$ EPCs represent a surrogate indicator for angiogenesis and response to antitumor strategies based on its mobilization. ${ }^{12,13}$

Endostatin (ES), initially discovered in the supernatant of a murine hemangioendothelioma cell line, was an inhibitor of endothelial cell proliferation in vitro and a robust angiogenesis inhibitor in vivo. ${ }^{14}$ In addition, gene therapy of ES for 
hepatoma via inhibiting tumor angiogenesis provided a promising result. ${ }^{15,16}$ However, there is no direct tumorkilling effect, which is a major drawback of these approaches.

Cytosine deaminase (CD) is a suicide enzyme capable of converting non-toxic 5-fluorocytosine (5-FC) into a cytotoxic 5-fluorouracil (5-FU), which is widely used as a chemotherapeutic agent. ${ }^{17}$ Several clinical trials have demonstrated that $\mathrm{CD}$ has the capability to kill hepatoma cells. $^{18,19}$ In this study, the therapeutic effect of EPCs armed with $\mathrm{CD}$ and ES fusion gene were studied in mouse hepatoma models and the feasibility of this technique was evaluated as a potential therapeutic approach for hepatoma.

\section{Materials and Methods EPC Culture}

Animal experimental procedure was approved by the Institutional Animal Care and Use Committee of Zhejiang University School of Medicine [No. SYXK (Zhejiang)2019-0012]. The EPCs were generated from the cardiac blood of male BALB/c nude mice, aged 6 weeks, provided by the Experimental Animal Center, Zhejiang University School of Medicine. The fresh blood withdrawn from the heart of the mice was heparinized and diluted with phosphate-buffered saline (PBS). Then, the monocyte layer was selected by density centrifugation and resuspended in microvascular growth medium-2 (EGM-2 MV; Cambrex, Walkersville, MD, USA) supplemented with 10\% fetal bovine serum (FBS; SAFC Biosciences, St. Louis, MO, USA). Mononuclear cells (MNCs) were cultured in a $25 \mathrm{~cm}^{3}$ culture flask with standard culture medium at $37{ }^{\circ} \mathrm{C}$ with $5 \% \mathrm{CO}_{2}$.

\section{In vitro Transfection of EPCs}

Lentiviral vectors (LVs) containing CD and ES fusion gene were constructed as described previously. ${ }^{20}$ The LVs were constructed by transfection of $293 \mathrm{~T}$ cells. The medium was changed after $24 \mathrm{~h}$ post-transfection. The conditioned medium collected after $48 \mathrm{~h}$ was centrifuged at $3000 \mathrm{rpm}$ for 15 min and filter sterilized through $0.45 \mu \mathrm{m}$ filters, followed by centrifugation at $50,000 \times g$ for $90 \mathrm{~min}$. The samples were diluted in DMEM and stored at $-80^{\circ} \mathrm{C}$.

For infection, EPCs were plated $24 \mathrm{~h}$ before infection with $\mathrm{LV}-\mathrm{CD} / \mathrm{ES}$ (MOI=5, 25, 50, $100 \mathrm{pfu} /$ cell) in DMEM with $10 \% \mathrm{FBS}$ for 48,72 , and $96 \mathrm{~h}$ at $37{ }^{\circ} \mathrm{C}$ in a $\mathrm{CO}_{2}$ incubator. The transfection efficiency of EPCs was assessed using a fluorescence microscope. Quantitative real-time PCR was performed to detect the mRNA levels of $\mathrm{CD}$ and ES genes. Western blot analysis was performed to detect the protein concentrations of supernatants.

\section{Proliferation Assay}

An MTT-based colorimetric proliferation assay (Sigma, St. Louis, MO, USA) was employed to test the killing effect of the EPCs transfected with LV-CD/ES. Trypsinized and resuspended EPCs $\left(3 \times 10^{4}\right.$ cells $\left./ \mathrm{mL}\right)$ were plated in the fibronectin-coated 96-well plates $\left(3 \times 10^{3} /\right.$ well $)$ at $37{ }^{\circ} \mathrm{C}$ for $24 \mathrm{~h}$. A volume of $10 \mu \mathrm{L}$ MTT reagent was added to each well after washing with PBS, followed by incubation at $37{ }^{\circ} \mathrm{C}$ for an additional $3-4 \mathrm{~h}$. The $\mathrm{OD}$ values were measured at $492 \mathrm{~nm}$ using an enzyme-linked immunosorbent detector (Thermo Scientific, Waltham, MA, USA).

\section{Migration Assay}

The chemotactic motility of the transfected EPCs was tested using Transwell chamber (Corning, USA) assay. A density of $1 \times 10^{5}$ cells, including EPCs, EPCs transfected with LVs, and EPCs transfected with LV-CD/ES were seeded in the upper chamber wells. The EGM-2 medium containing $10 \%$ FBS was placed in the lower chamber as a chemoattractant. The chamber was incubated at $37{ }^{\circ} \mathrm{C}$ for $48 \mathrm{~h}$. After the non-migrating cells were discarded and upper wells washed with PBS, the filters were scraped with cotton swabs, and the cells were fixed in $4 \%$ paraformaldehyde and stained with $0.5 \%$ crystal violet. The migrated cells were viewed and enumerated under an inverted microscope (Olympus).

\section{Tumor Models and in vivo Antitumor Experiments}

BALB/c nude mouse hepatoma model, aged 6 weeks, was provided and housed in specific-pathogen-free (SPF) laminar air flow rooms at the Experimental Animal Center, Zhejiang University School of Medicine. Animals had free access to food and water. All experiments in this study were performed in agreement with the experimental animal ethical standards of Zhejiang University School of Medicine.

The mouse tumor model of human HepG2 liver cancer was used in this study. HepG2 cells were purchased from Anti-Cancer Biotech Co., Ltd. (Beijing, China). An equivalent of $2 \times 10^{6}$ HepG2 cells $(1 \mathrm{~mL})$ suspension was injected into the subcutaneous tissues of the mice. Two 
weeks post-implantation, a tumor of $3 \mathrm{~cm}$ was stripped from the subcutaneous tissues of the mice. The tumor was sliced into $1 \mathrm{~mm}^{3}$ fragments and injected into the mouse liver.

In order to assess the antitumor effects of CD/ES-EPCs in vivo, the mice were randomly assigned to five groups ( $n=15$ /group) as follows: group 1 normal mice without tumor, group 2 hepG2-bearing mice without treatment, group 3 hepG2-bearing mice with EPCs (injection via tail vein, $1 \times 10^{6}$ cells/mouse), group 4 hepG2-bearing mice with $\mathrm{CD} / \mathrm{ES}$ (injection via tail vein, $1 \times 10^{8} \mathrm{TU} / \mathrm{mL}$ ), and group 5 hepG2-bearing mice with $\mathrm{CD} / \mathrm{ES}-\mathrm{EPCs}$ (injection via tail vein, $1 \times 10^{6}$ cells/mouse). Next, 5-FC $(500 \mathrm{mg} / \mathrm{kg} / \mathrm{d})$ was injected intraperitoneally into groups 4 and 5 two times daily after the last inoculation.

The Biospec 70/20USR 7.0T MRI (Bruker, Germany) was used for the detection of hepatoma in mice after in vivo transfection. The scanning parameters of MRI were as described previously: ${ }^{20}$ T2WI scan; RARE sequence, TR/TE: $1300 \mathrm{~ms} / 7.5 \mathrm{~ms}$; slice thickness $1 \mathrm{~mm}$; images in acquisition 16; FOV $3.5 \mathrm{~mm} \times 3.5 \mathrm{~mm}$; flip angle $160^{\circ}$; matrix $256 \times 256$. The volumes of the tumors were calculated by assessing the T2WI images using Render ToolKit 1.0.The mice in the study were sacrificed at the end of experiment.

\section{Immunohistochemistry and Histological Staining}

The tumors were excised and fixed in 10\% formalin overnight. The tissues were dehydrated, embedded in paraffin, and sectioned into 5- $\mu \mathrm{m}$ slices for immunohistochemistry staining. The slides were blocked for the non-specific antibody, and subsequently, probed with primary antibodies overnight at room temperature. After rinsing with PBS, the slides were incubated with secondary antibodies for 60 min at ambient temperature and washed again with PBS. The immunostained sections from peripheral and central regions of each tumor were imaged using a fluorescent microscope (Olympus, Center Valley, PA, USA). A minimum of 5 fields/section and 5 sections/tumor sample were analyzed.

\section{In vivo Apoptotic TUNEL (Terminal Deoxynucleotidyl Transferase Mediated dUTP Nick End Labeling)}

For apoptotic assay in vivo, tumor tissues were embedded in paraffin blocks. The slides with the sections were washed with PBS and incubated with TUNEL reaction mixture (Roche Diagnostics, USA) at $37{ }^{\circ} \mathrm{C}$ for $30 \mathrm{~min}$ according to the manufacturer's protocol. The apoptotic cells of positive TUNEL staining were counted in five random fields under a fluorescent microscope.

\section{Statistical Analysis}

Statistical analyses were performed using the $\operatorname{SPSS}^{\circledR}$ statistical package, version 25.0 (SPSS Inc., Chicago, IL, USA) for Windows ${ }^{\circledR}$. Data for continuous variables are presented as means \pm standard deviation (SD). The differences between the study and control groups were determined by the Mann-Whitney $U$-test or the unpaired Student's $t$-test where appropriate. $P$-values $<0.05$ were considered as statistically significant.

\section{Results}

\section{In vitro Biological Function Assay of CD/ES}

To inspect the possibility of CD/ES gene for cancer therapy, the mRNA levels of ES and CD genes were confirmed by quantitative real-time PCR. The protein expression of ES was confirmed by Western blot analysis. The results showed a significant increase in mRNA levels (Figure 1) and protein concentrations (Figure 2) in the groups transfected with $\mathrm{CD} /$ $\mathrm{ES}$ as compared to groups treated with EPCs or EPCs $+\mathrm{LV}$.

To investigate the 5-FC prodrug-converting enzyme activity of $\mathrm{CD} / \mathrm{ES}$, the high-performance liquid chromatography (HPLC) assay was employed to measure the 5-FU concentrations of supernatants. This result showed a marked increase in 5-FU concentrations in the groups transfected with $\mathrm{CD} / \mathrm{ES}$ (Figure 3).

Furthermore, to assess the antiangiogenic activity of $\mathrm{CD} / \mathrm{ES}$, we performed proliferation and migration assays to inspect the effects of the gene. The results showed a significant decrease in cell proliferation (Figure 4) and the number of migrated cells (Figure 5) in the groups transfected with $\mathrm{CD} / \mathrm{ES}$ as compared to groups treated with EPCs or EPCs+LV.

\section{In vivo Tail Vein-Injected CD/ES-EPCs Suppressed the Tumor Growth}

After transplantation of CD/ES-EPCs plus 5-FC into the mouse with hepatoma, the tumor volumes among five groups ( $n=15 /$ group) were observed by MRI. The results showed that the tumor volumes in the group transfected with $\mathrm{CD} / \mathrm{ES}$-EPCs plus 5-FC were smaller than that in the 

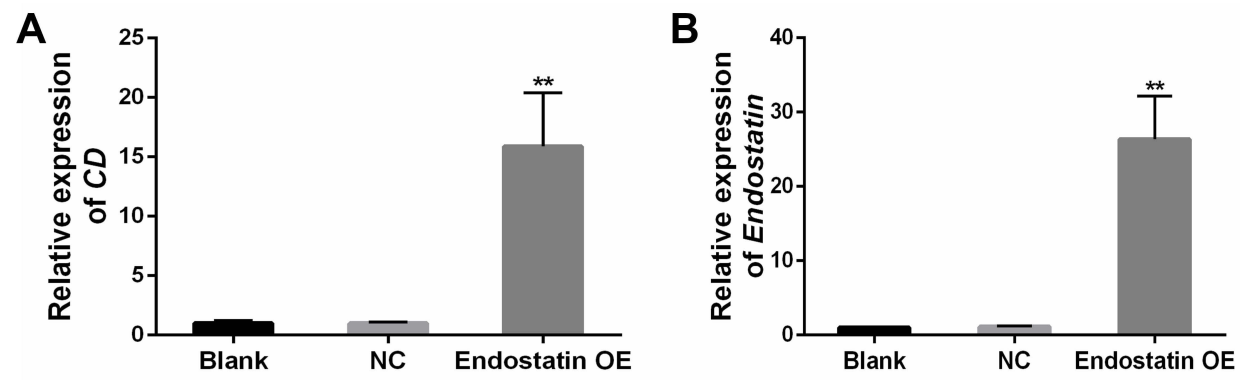

Figure I mRNA levels of CD and ES genes. (A) CD gene. (B) ES gene. Blank: EPCs; NC: EPCs transfected with LV; Endostatin OE: EPCs transfected with LV-CD/ES $(* * P<0.01$ vs $N C)$.

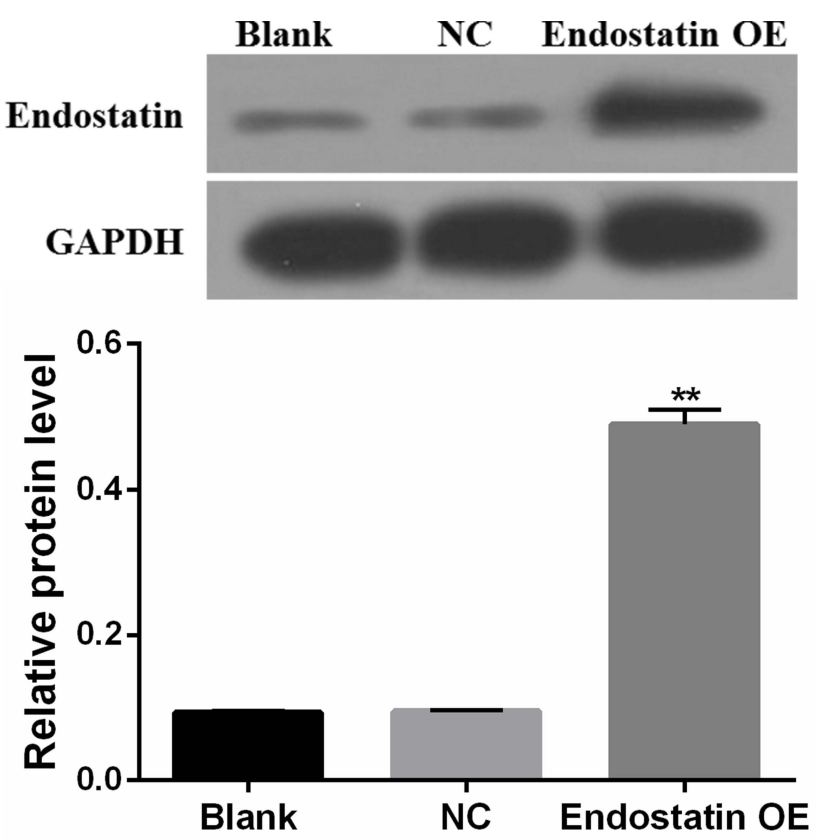

Figure 2 Western blot analysis of endostatin. Blank: EPCs; NC: EPCs transfected with LV; Endostatin OE: EPCs transfected with LV-CD/ES (**P<0.01 vs NC).

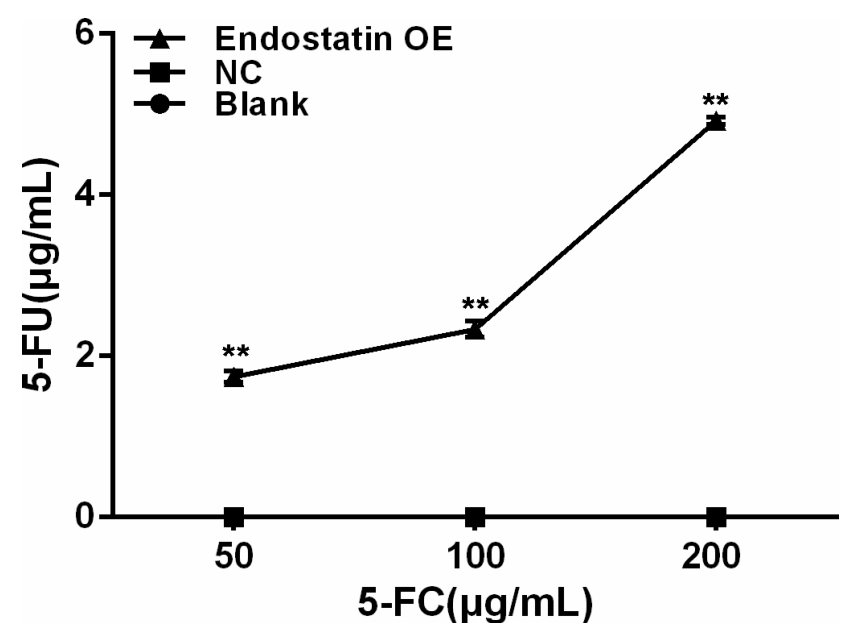

Figure 3 5-FU concentrations of supernatants. Blank: EPCs; NC: EPCs transfected with LV; Endostatin OE: EPCs transfected with LV-CD/ES (**P<0.0I vs NC).

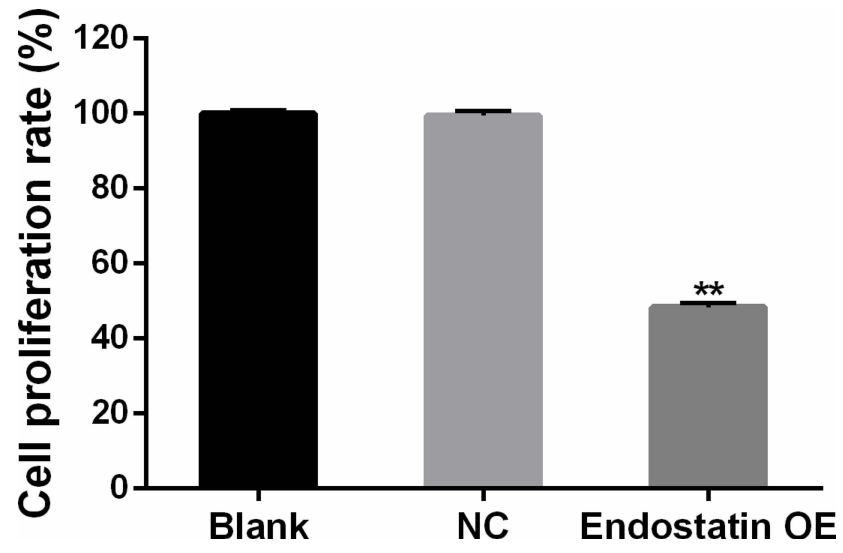

Figure 4 Cell proliferation among the three groups. Blank: EPCs; NC: EPCs transfected with LV; Endostatin OE: EPCs transfected with LV-CD/ES $(* * P<0.01$ vs $\mathrm{NC}$ ).

other groups receiving other treatments after 2 weeks posttransfection (Figure 6).

\section{CD/ES-EPCs Inhibited Angiogenesis and Induced Tumor Cell Apoptosis}

VEGF immunohistochemistry staining of the tumor tissues showed that the positive rate of VEGF in the tumor tissues was greatly reduced in the ES/CD-EPCs treated group when compared to the other treatment groups, (Figure 7). Similarly, the CD31-positive staining showed that the number of endothelial cells in the tumor tissues was greatly decreased in the ES/CD-EPCs treated group compared to the other treatment groups, further supporting the tumor suppression results (Figure 8). The number of apoptotic tumor cells detected by the TUNEL assay in the group treated with CD/ES-EPCs was significantly higher than that in other groups (Figure 9).

\section{Discussion}

$\mathrm{CD}$ is a therapeutic suicide gene for tumors and can convert the non-toxic 5-FC into cytotoxic 5-FU, which is a standard 

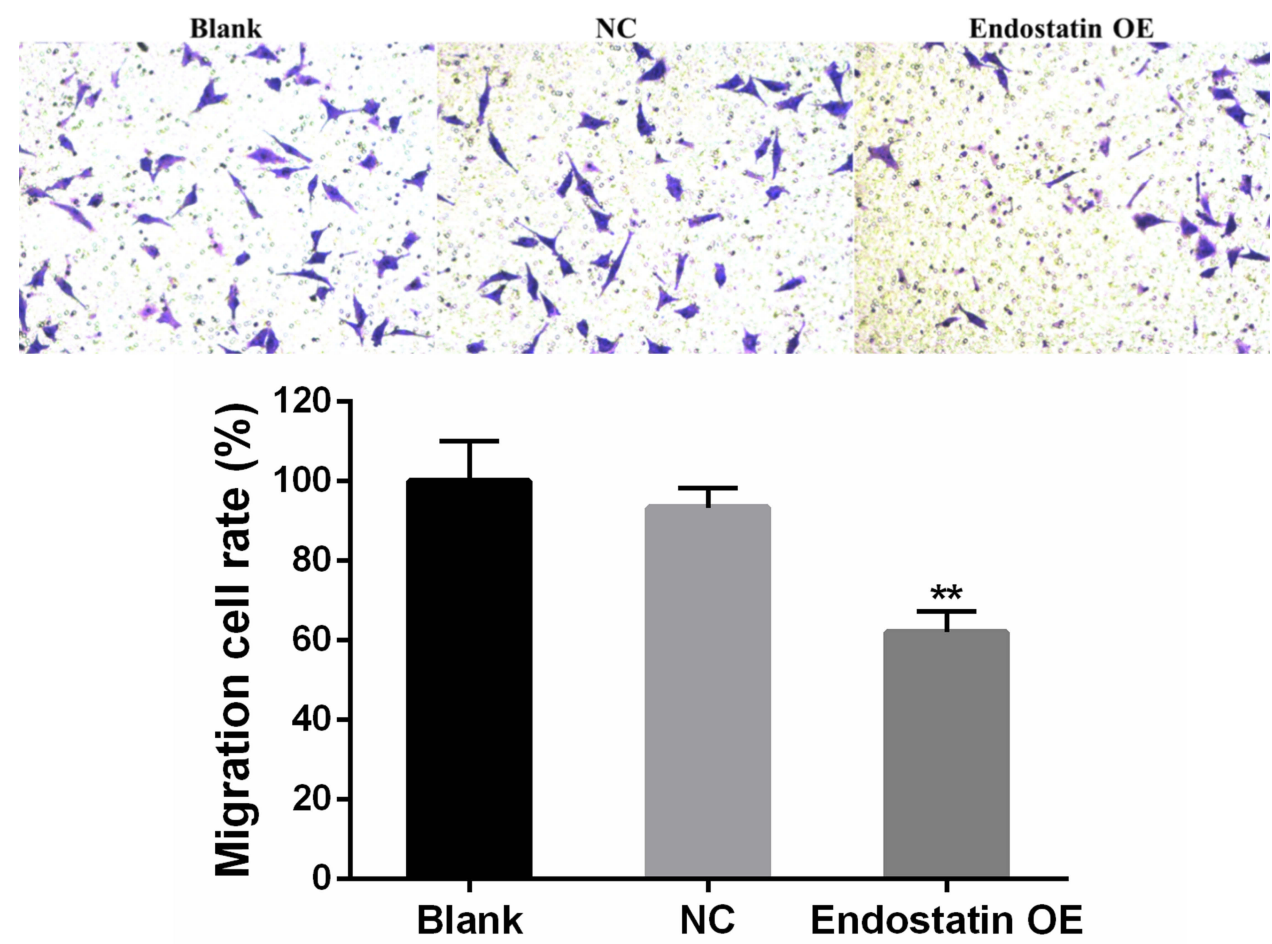

Figure 5 Cell migration among the three groups $(\times 200)$. Blank: EPCs; NC: EPCs transfected with LV; Endostatin OE: EPCs transfected with LV-CD/ES (**P<0.0I vs NC).

A

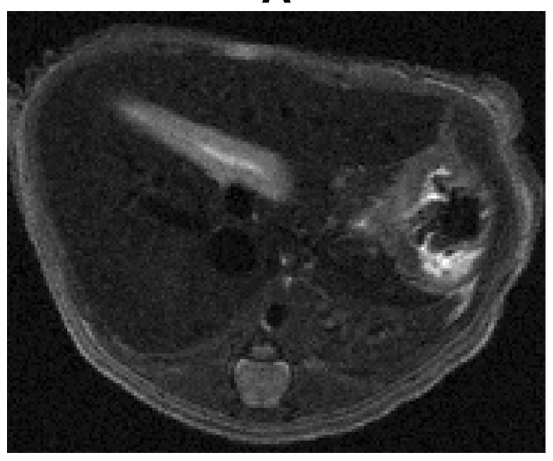

D

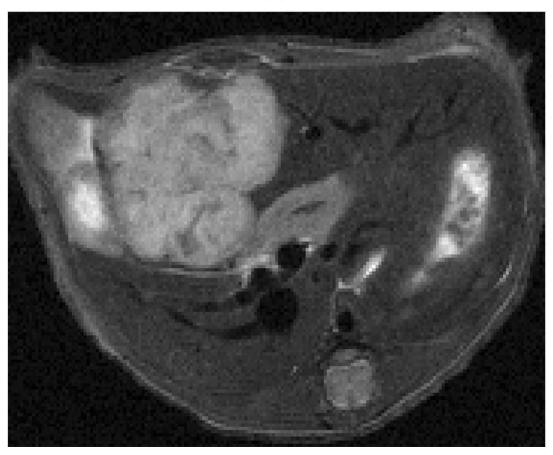

B

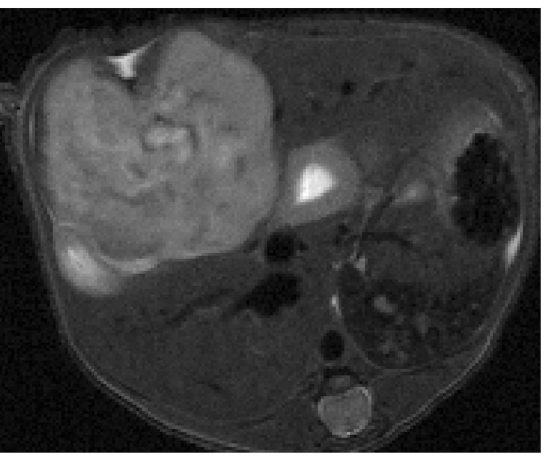

E

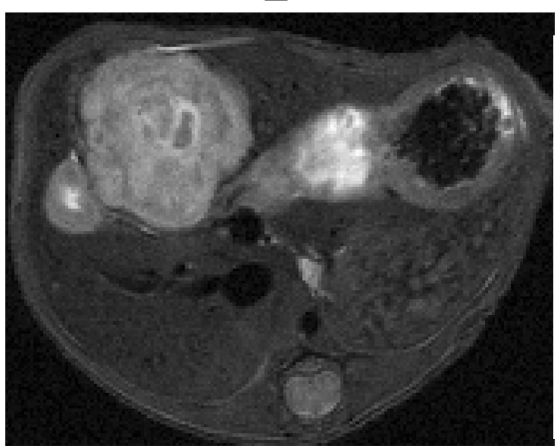

C

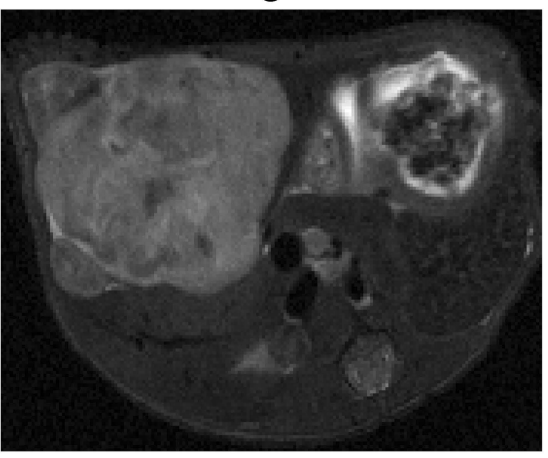

F

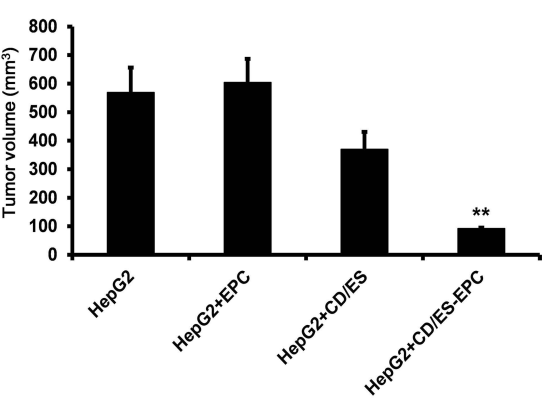

Figure 6 Tumor volumes assessed by MRI after cell transplantation. (A) Control, (B) HepG2, (C) HepG2+EPC, (D) HepG2+CD/ES, (E) HepG2+CD/ES-EPC, and (F) statistical analysis of tumor volumes $(* * P<0.01$ vs other groups). 


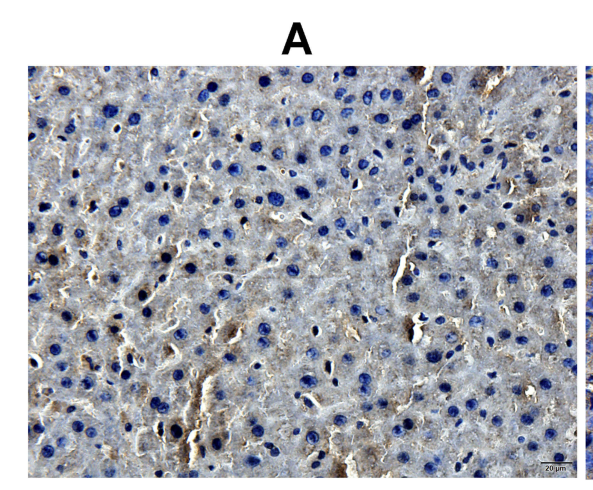

D

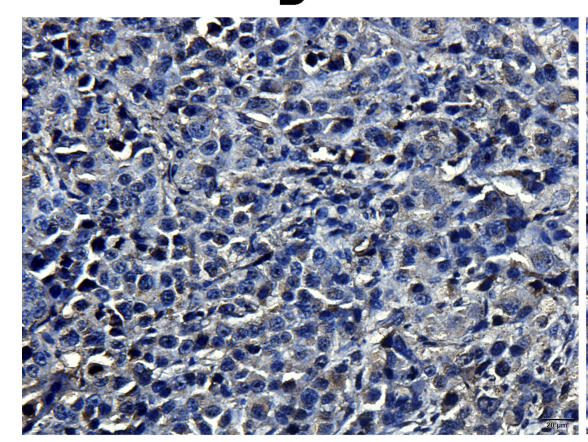

B

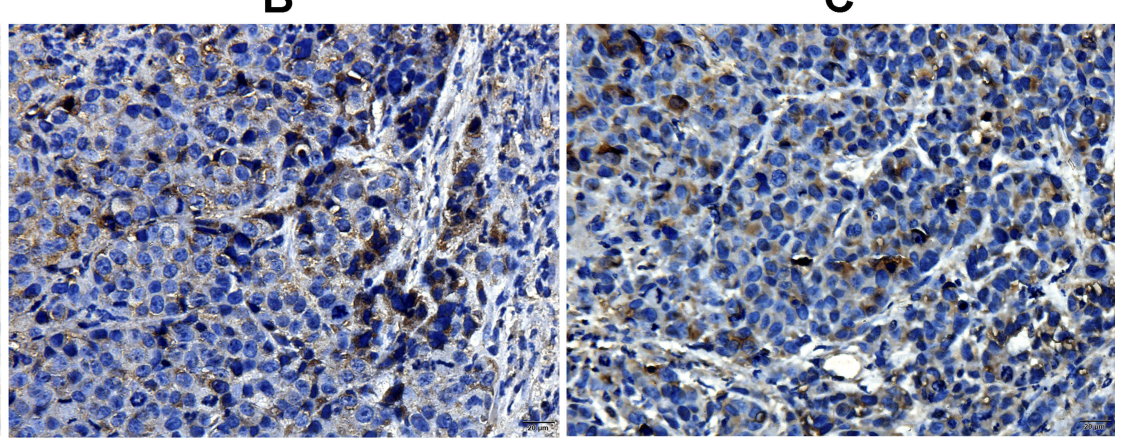

E

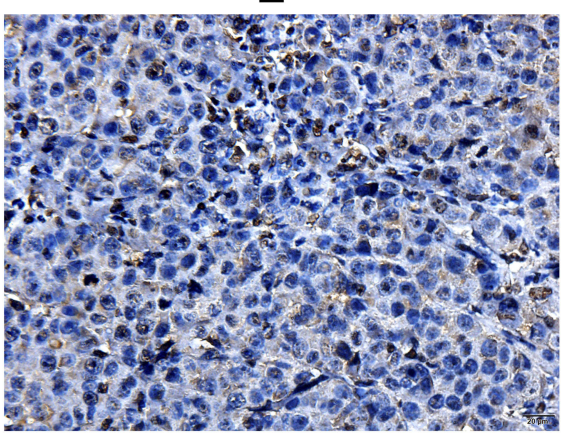

$\mathbf{F}$

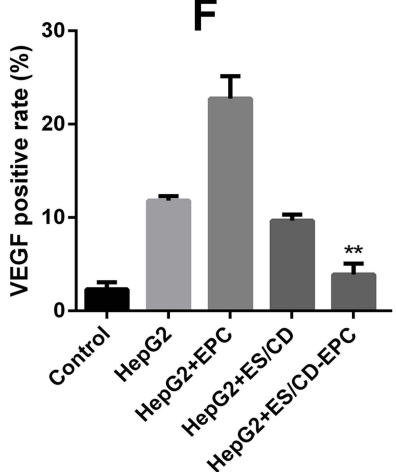

Figure 7 VEGF staining after cell transplantation. (A) Control, (B) HepG2, (C) HepG2+EPC, (D) HepG2+CD/ES, (E) HepG2+CD/ES-EPC, and (F) statistical analysis of VEGF-positive rate $(* * P<0.01$ vs other treatment groups).

A

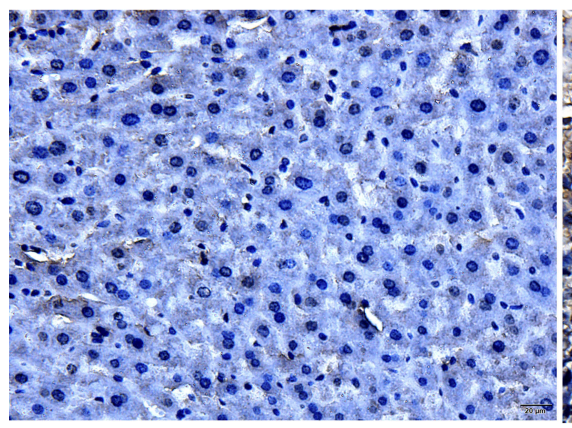

D

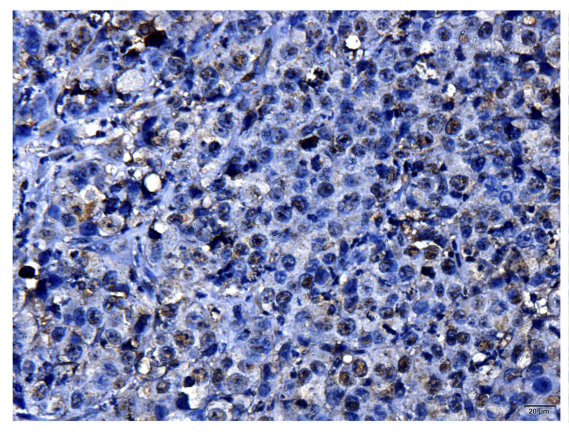

B

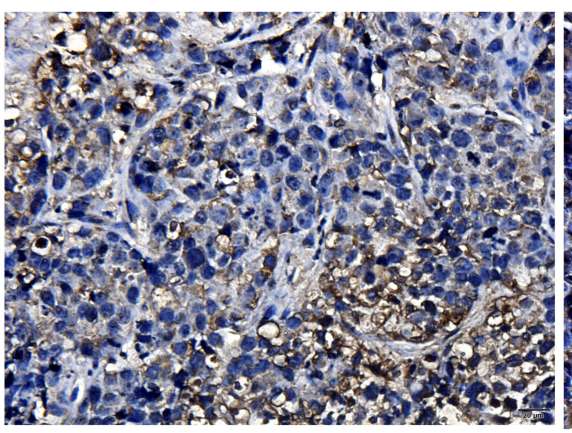

E

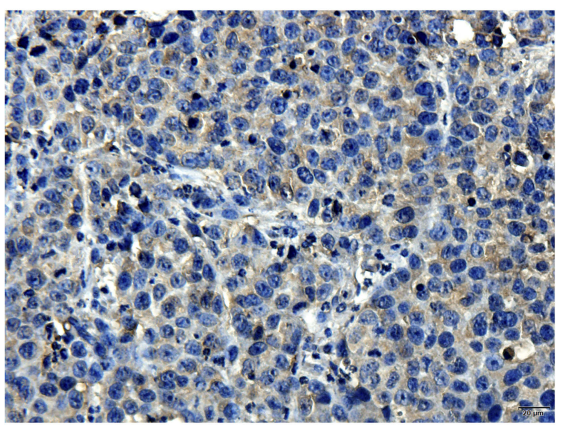

C

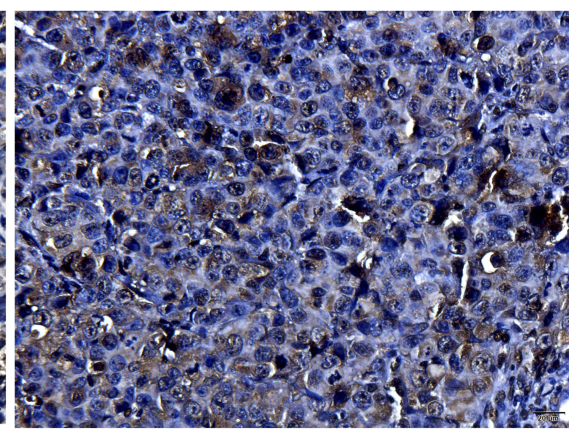

$\mathbf{F}$

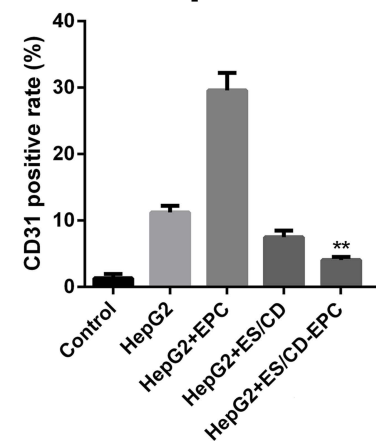

Figure 8 CD31-positive staining after cell transplantation. (A) Control, (B) HepG2, (C) HepG2+EPC, (D) HepG2+CD/ES, (E) HepG2+CD/ES-EPC, and (F) statistical analysis of $\mathrm{CD} 3 \mathrm{I}$-positive rate $(* * \mathrm{P}<0.01$ vs other treatment groups). 

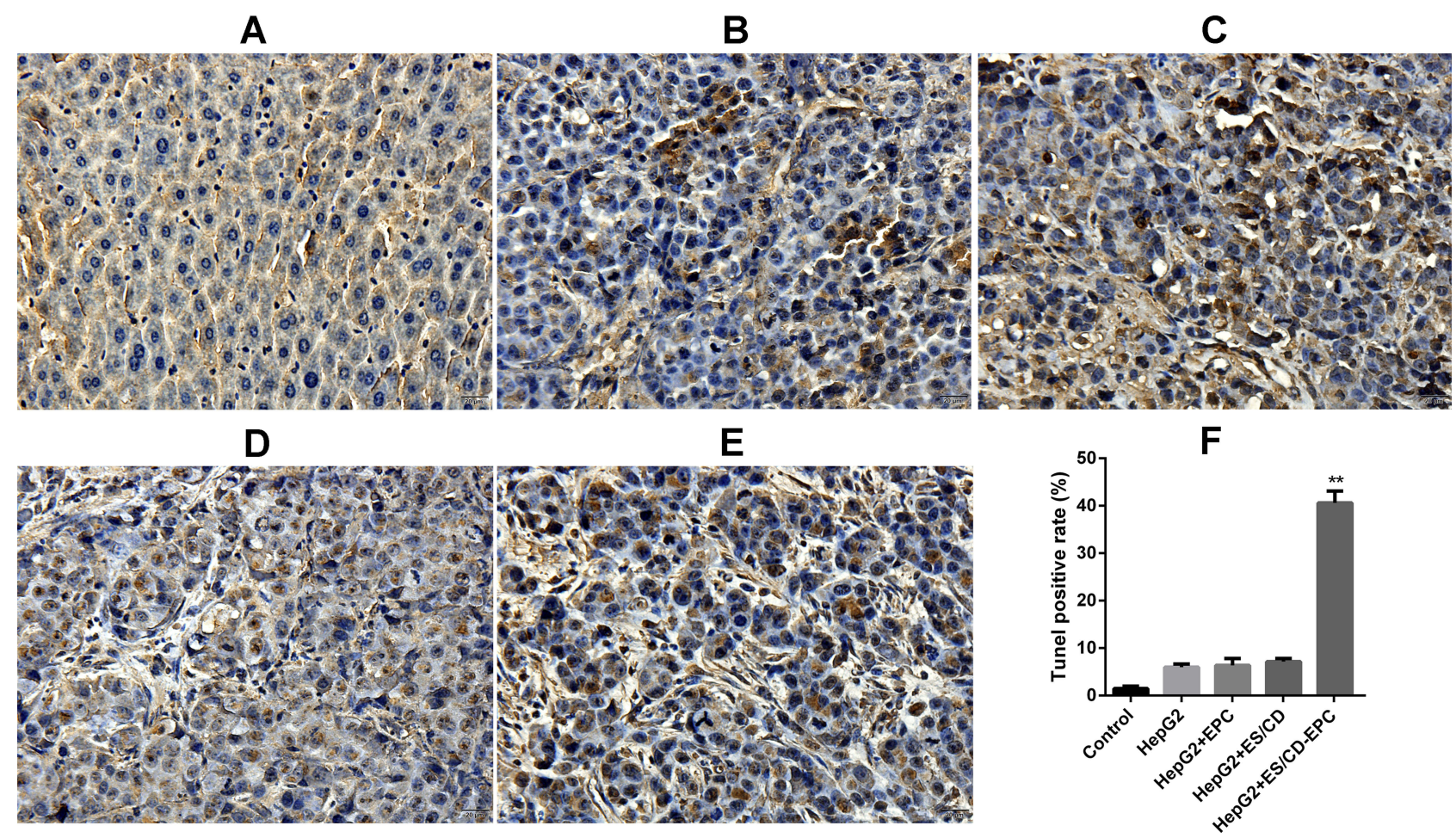

Figure 9 Apoptosis of tumor cells after cell transplantation. (A) Control, (B) HepG2, (C) HepG2+EPC, (D) HepG2+CD/ES, (E) HepG2+CD/ES-EPC, and (F) statistical analysis of TUNEL-positive rate ( $* * P<0.01$ vs other groups).

chemotherapeutic agent that inhibits RNA and DNA synthesis. ${ }^{17}$ Metronomic chemotherapy suppressed the endothelial cell growth and induces apoptosis of tumor cell or cell growth arrest. ${ }^{21}$ Similarly, ES can up-regulate several antiangiogenic genes, such as maspin, AT3, kininogen, vasostatin, and THBS-1. ES can also down-regulate several proangiogenic signaling pathway components, such as nuclear factor- $\beta$, activator protein-1, activators of transcription and signal transducers. ${ }^{22}$ Recent studies have shown that ES suppresses the growth of tumor tissues in the tumorbearing animal models. ${ }^{20,23,24}$ The CD and ES genes act synergistically to effectuate the tumor-killing effect as well as inhibiting the tumor angiogenesis. In this study, the in vitro transfection of the CD/ES genes into the EPCs was confirmed by quantitative real-time PCR, Western blot analysis, and proliferation and migration assays.

Some studies have shown that the EPCs, predominantly homing to tumor tissues, might be used as cellular carriers for delivering the therapeutic signals to tumor sites. Varma et al used EPCs as part of a gene carrier/ delivery system for glioma therapy and imaging probes. The study by Bonfim-Silva et al showed that the BMderived EPCs are mobilized into the bloodstream, and specifically migrate to the tumor sites in vivo. To monitor the transplanted EPCs and verify the effectiveness of the EPCs as cellular carriers for tumor therapy, several noninvasive in vivo tracking imaging techniques such as MRI, nuclear medicine, and optical imaging have been used. ${ }^{25,26}$ After transplantation of the intravenously administered CD/ES-EPCs into the mouse hepatoma models, MRI was used for in vivo observation of the volume changes of the tumors. In addition, the current in vivo study showed that the tumor volumes were reduced in the CD/ES-EPCs group as compared to the other treatment groups. Moreover, the VEGF staining and TUNEL test indirectly demonstrated that $\mathrm{CD} / \mathrm{ES}-\mathrm{EPC}$ were present in the tumor tissues. The VEGF-positive rate was lowest and the number of apoptosis cells was highest in the CD/ES-EPCs group. This result illustrated that $\mathrm{CD} / \mathrm{ES}-\mathrm{EPCs}$ promote apoptosis of tumor cells and inhibit the tumor angiogenesis.

\section{Conclusion}

This study offers a novel treatment strategy for inhibiting the tumor growth by CD/ES-transfected EPCs targeting the tumor sites via vein grafting that can serve as a potent therapeutic option for the treatment of hepatoma after further investigation by clinical trials. 


\section{Ethical Statement}

Animal experimental protocols were approved by the Institutional Animal Care and Use Committee of Zhejiang University School of Medicine (approval ID: SYXK(ZHE)2019-0012).

\section{Acknowledgments}

The present work was funded by Zhejiang Provincial Natural Science Foundation of China (Grant No. LZ18H180001), National Natural Science Foundation of China (Grant No. 81371658 and 81971713), National S\&T Major Project of China (NO. 2018ZX10301201), Grant from Health Commission of Zhejiang Province (JBZX202004), Research Unit of Collaborative Diagnosis and Treatment For Hepatobiliary and Pancreatic Cancer, Chinese Academy of Medical Sciences (2019RU019), The Key Research Development Program of Zhejiang province (Grant No. 2018C03018), Key Science and Technology Program of Zhejiang province (No. WKJ-ZJ1923) and National Key R\&D Program of China (No. 2017YFC0114102).

All authors thank Professor Xinmei Chen from Shandong University of Traditional Chinese Medicine, who is currently a visiting scholar of Harvard University, USA, for her kind proofreading.

\section{Disclosure}

The authors report no conflicts of interest in this work.

\section{References}

1. Bosetti C, Turati F, La Vecchia C. Hepatocellular carcinoma epidemiology. Best Pract Res Clin Gastroenterol. 2014;28(5):753-770. doi:10.1016/j.bpg.2014.08.007

2. Maluccio M, Covey A. Recent progress in understanding, diagnosing, and treating hepatocellular carcinoma. CA Cancer J Clin. 2012;62 (6):394-399. doi:10.3322/caac.21161

3. Zhang S, Yue M, Shu R, et al. Recent advances in the management of hepatocellular carcinoma. J BUON. 2016;21:307-311.

4. Zhang W, Jiang L, Yan L, et al. Radiofrequency ablation for HCC patients with multifocal tumours meeting the Milan criteria: a singlecentre experience. Dig Liver Dis. 2016;48:1485-1491. doi:10.1016/j. dld.2016.07.018

5. Wei WX, Yang ZS, Lu LH, et al. Long-term survival after partial hepatectomy for sub-stage patients with intermediate stage hepatocellular carcinoma. Int $J$ Surg. 2018;56:256-263. doi:10.1016/j. ijsu.2018.06.020

6. Zhong C, Zhang YF, Huang JH, et al. Comparison of hepatic resection and transarterial chemoembolization for UICC stage T3 hepatocellular carcinoma: a propensity score matching study. BMC Cancer. 2018;18:643. doi:10.1186/s12885-018-4557-5

7. Greten TF, Duffy AG, Korangy F. Hepatocellular carcinoma from an immunologic perspective. Clin Cancer Res. 2013;19:6678-6685. doi:10.1158/1078-0432.CCR-13-1721
8. Varma NR, Janic B, Iskander AS, et al. Endothelial progenitor cells (EPCs) as gene carrier system for rat model of human glioma. PLoS One. 2012;7:e30310. doi:10.1371/journal.pone.0030310

9. Khakoo AY, Finkel T. Endothelial progenitor cells. Annu Rev Med. 2005;56:79-101. doi:10.1146/annurev.med.56.090203.104149

10. Zhao X, Liu HQ, Li J, et al. Endothelial progenitor cells promote tumor growth and progression by enhancing new vessel formation. Oncol Lett. 2016;12:793-799. doi:10.3892/ol.2016.4733

11. Bonfim-Silva R, Souza LE, Melo FU, et al. Bone marrow-derived cells are recruited by the melanoma tumor with endothelial cells contributing to tumor vasculature. Clin Transl Oncol. 2017;19:125133. doi:10.1007/s12094-016-1515-z

12. Laurenzana A, Biagioni A, D'Alessio S, et al. Melanoma cell therapy: endothelial progenitor cells as shuttle of the MMP12 uPARdegrading enzyme. Oncotarget. 2014;5:3711-3727. doi:10.18632/ oncotarget. 1987

13. Wang G, Zhang L, Zhou Y, et al. KAI1/CD82 genetically engineered endothelial progenitor cells inhibit metastasis of human nasopharyngeal carcinoma in a mouse model. Med Sci Monit. 2018;24:31463152. doi:10.12659/MSM.907219

14. O'Reilly MS, Boehm T, Shing Y, et al. Endostatin: an endogenous inhibitor of angiogenesis and tumor growth. Cell. 1997;88:277-285. doi:10.1016/S0092-8674(00)81848-6

15. Ren Z, Wang Y, Jiang W, et al. Anti-tumor effect of a novel soluble recombinant human endostatin: administered as a single agent or in combination with chemotherapy agents in mouse tumor models. PLoS One. 2014;9:e107823. doi:10.1371/journal.pone.0107823

16. Yan M, Dongmei B, Jingjing Z, et al. Antitumor activities of livertargeting peptide modified recombinant human endostatin in BALB/ c-nu mice with hepatocellular carcinoma. Sci Rep. 2017;7:14074. doi:10.1038/s41598-017-14320-0

17. Mesa-Pereira B, Medina C, Camacho EM, et al. Improved cytotoxic effects of Salmonella-producing cytosine deaminase in tumour cells. Microb Biotechnol. 2015;8:169-176. doi:10.1111/1751-7915.12153

18. Torimura $\mathrm{T}$, Ueno $\mathrm{T}$, Taniguchi $\mathrm{E}$, et al. Interaction of endothelial progenitor cells expressing cytosine deaminase in tumor tissues and 5 -fluorocytosine administration suppresses growth of 5-fluorouracil sensitive liver cancer in mice. Cancer Sci. 2012;103:542-548. doi:10.1111/j.1349-7006.2011.02182.x

19. Chen R, Yu H, An YL, et al. Genetic immunotherapy for hepatocellular carcinoma by endothelial progenitor cells armed with cytosine deaminase. J Biomed Nanotechnol. 2014;10:271-277. doi:10.1166/ jbn.2014.1766

20. Chen R, Yu H, An YL, et al. Endothelial progenitor cells combined with cytosine deaminase-endostatin for suppression of liver carcinoma. $J$ Biomed Nanotechnol. 2016;12:1174-1182. doi:10.1166/jbn.2016.2261

21. Kerbel RS, Kamen BA. The anti-angiogenic basis of metronomic chemotherapy. Nat Rev Cancer. 2004;4(6):423-436. doi:10.1038/ $\operatorname{nrc} 1369$

22. Abdollahi A, Hahnfeldt P, Maercker C, et al. Endostatin's antiangiogenic signaling network. Mol Cell. 2004;13:649-663. doi:10.1016/ S1097-2765(04)00102-9

23. Jia Y, Liu M, Huang W, et al. Recombinant human endostatin endostar inhibits tumor growth and metastasis in a mouse xenograft model of colon cancer. Pathol Oncol Res. 2012;18:315-323. doi:10.1007/ s12253-011-9447-y

24. Shan YF, Fang YF, Wang XQ, et al. Experimental studies on treatment of pancreatic cancer with double-regulated duplicative adenovirus AdTPHre-hEndo carrying human endostatin gene. Pancreatology. 2013;13:393-400. doi:10.1016/j.pan.2013.05.012

25. Ngen EJ, Kato Y, Artemov D. Direct cell labeling to image transplanted stem cells in real time using a dual-contrast MRI technique. Curr Protoc Stem Cell Biol. 2017;42:5A.10.1-5A.10.19. doi:10.1002/cpsc.33

26. Zheng Y, Huang J, Zhu T, et al. Stem cell tracking technologies for neurological regenerative medicine purposes. Stem Cells Int. 2017;2017:2934149. doi:10.1155/2017/2934149 


\section{Publish your work in this journal}

Cancer Management and Research is an international, peer-reviewed open access journal focusing on cancer research and the optimal use of preventative and integrated treatment interventions to achieve improved outcomes, enhanced survival and quality of life for the cancer patient.

The manuscript management system is completely online and includes a very quick and fair peer-review system, which is all easy to use. Visit http://www.dovepress.com/testimonials.php to read real quotes from published authors. 